\title{
s-PROCESS ENRICHMENT IN LOW-MASS AGB STARS
}

\author{
R.Gallino
}

Istituto di Fisica Generale dell'Università, Torino, Italy

\begin{abstract}
After a brief description of the developments of the theory of s-process nucleosynthesis, the difficulties recently encountered in envisaging reliable astrophysical conditions for obtaining a solar-system distribution of s-isotopes are discussed. In particular, while the reaction ${ }^{22} \mathrm{Ne}(\alpha, n){ }^{25} \mathrm{Mg}$ may account for the nucleosynthesis of the weak $s-$ component in massive stars, it fails to reproduce the main s-component in intermediate mass stars. The efficiency of the alternative reaction ${ }^{13} \mathrm{C}(\alpha, n){ }^{16} \mathrm{O}$ occurring in low mass stars during recurring thermal instabilities of the He shell is then analyzed. It is shown that, contrary to previous expectations, the ${ }^{13} \mathrm{C}$ source well reproduces the main component, provided that realistic physical conditions are assumed for the temporal behaviour of the pulse and the effect of the light $n$-absorbers (especially ${ }^{12} \mathrm{C}$ ) is properly taken into account. The results satisfactorily compare with the constraints of the classical s-analysis. Key observational evidences also appear to be in agreement with this scenario.
\end{abstract}

\section{INTRODUCTION}

The analysis of Clayton et al. (1961) and Seeger et al. (1965) of the products $\sigma_{i} N_{s, i}$ of $s$-only isotopes, where $\sigma_{i}$ is the $n$-capture cross section of a given isotope and $N_{s, i}$ its solar-system abundance, demonstrated that the s-isotopes cannot be reproduced by a unique neutron irradiation, but more likely by an exponential distribution of neutron exposures

$\rho(\tau) \mathrm{d} \tau=G e^{\left(-\tau / \tau_{0}\right)} d \tau$

where $\rho(\tau) \mathrm{d} \tau$ represents the number of iron seeds irradiated with a neutron exposure between $\tau$ and $\tau+d \tau$. The neutron exposure $\tau=\int N_{n} v_{T} d t$ is the time integrated product of neutron density and thermal velocity for a given irradiation. The fitting constant $\tau_{0}$ is called the neutron exposure parameter, and $G=$ $f N_{56} / \tau_{0}$, being $f$ the fraction of iron seed nuclei $N_{56}$ that suffered $n$-captures. Actually, as first suggested by Clayton and Rassbach (1967), and extensively discussed by Ward and Newman (1978), Käppeler et al. (1982), Beer (1986a,b), and Walter et al. (1986), at least two different distributions of neutron exposures are required: a weak component, which is responsible for the synthesis of the s-nuclei with $A \leq 90$, and a main component to account for the heavier isotopes; a third strong component is not excluded, in order to reproduce the heaviest $\mathrm{Pb}$ isotopes. For the weak, as well as for the strong component, a single irradiation appears more suitable to reproduce the data than an exponential distribution of neutron exposures (Beer, 1986b; Beer and Macklin, 1988b). Fig. 1 shows the best fit of the 


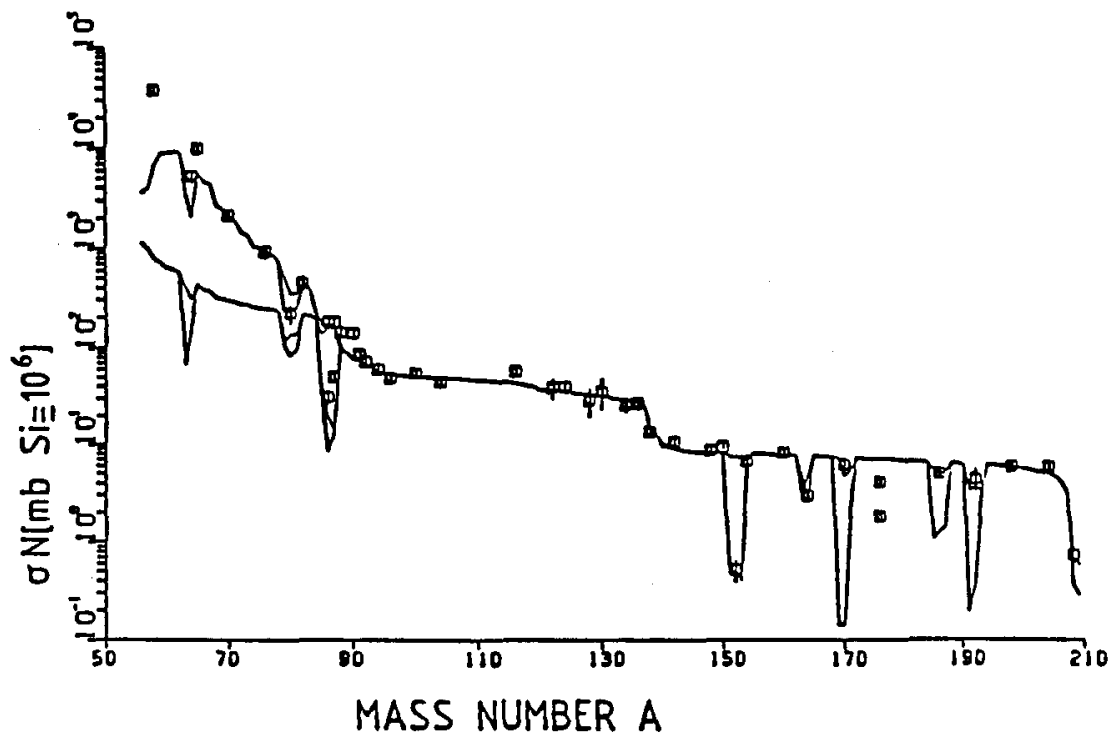

Fig. 1. Solar-system $\sigma N$ distribution of sonly isotopes fitted by a main pulsed scomponent. Below $A=90$ the weak s-component with a single irradiation is superimposed and the main component is shown separately. In the region of the heaviest isotopes the strong s component is effective. [From Beer and Macklin, 1988b].

Tabje 1

Parameters of the three s-process components.

\begin{tabular}{|c|c|c|c|c|c|}
\hline & \multicolumn{2}{|c|}{ Fraction of iron seeds (\%) } & \multicolumn{2}{|c|}{ Exposure to $\left(\mathrm{mb}^{-1}\right)$} & \multirow{2}{*}{$\frac{n_{\varepsilon}}{E E}$} \\
\hline & $s F$ & $E E$ & $S F$ & $E E$ & \\
\hline Main & - & $0.048 \pm 0.003$ & - & $0.30 \pm 0.01$ & $11.2 \pm 0.7$ \\
\hline Weak & 0.32 & 0.26 & 0.23 & $0.06 \pm 0.01$ & $1.4 \pm 0.4$ \\
\hline Strong & $0.910^{-4}$ & $1.2 \pm 0.710^{-4}$ & $Z 2.5$ & 26 & $2 נ 50$ \\
\hline
\end{tabular}

SF: single fiux s-process

EE: s-process with exponential exposure distribution

Data are from:

Beer (j966a,b), Bees et al. (1984), Walter et ul. (1986), Becr and Macklin (1956b). 
$\sigma N_{s}$ distribution, and Table 1 shows the characteristic parameters for the three components.

The classical analysis of s-processing can be undertaken independently of the true astrophysical sites and neutron sources. Concerning n-sources, for a long time the most promising ones are known to be the two reactions ${ }^{22} \mathrm{Ne}(\alpha, n)^{25} \mathrm{Mg}$ and ${ }^{13} \mathrm{C}(\alpha, n){ }^{16} \mathrm{O}$ (Cameron, 1955; Burbidge et al., 1957; Reeves, 1966). Both reactions are typical of He-burning environments. Large amounts of ${ }^{22} \mathrm{Ne}$ are expected from the chain of reactions ${ }^{14} \mathrm{~N}(\alpha, \gamma){ }^{18} \mathrm{~F}\left(\beta^{+} \nu\right)^{18} \mathrm{O}(\alpha, \gamma){ }^{22} \mathrm{Ne}$ that occurs at the very beginning of He-burning. Nevertheless, the ${ }^{22} \mathrm{Ne} n$-source requires rather high temperatures $\left(T \gtrsim 310^{8} \mathrm{~K}\right)$ to be activated. On the contrary, the ${ }^{13} \mathrm{C}$ source can easily take place at lower temperatures $\left(T \simeq 1.5 \quad 10^{8} \mathrm{~K}\right)$; more difficult is to envisage how sufficient amounts of ${ }^{13} \mathrm{C}$ should be present there. This requires some mixing process to dredge down a small amount of protons from the envelope into the ${ }^{12} \mathrm{C}$-rich region, in order to make the formation of ${ }^{13} \mathrm{C}$ possible through the chain ${ }^{12} \mathrm{C}(p, \gamma){ }^{13} \mathrm{~N}\left(\beta^{+} \nu\right){ }^{13} \mathrm{C}$, without further proceeding to the reaction $\left.{ }^{13} \mathrm{C}(p, \gamma)\right)^{14} \mathrm{~N}$. Actually, the lifetime of ${ }^{13} \mathrm{C}$ against $p$-captures being about 4 times less than that of ${ }^{12} \mathrm{C}$, the production of a certain fraction of ${ }^{14} \mathrm{~N}$ is unavoidable; this works against the synthesis of s-isotopes, since ${ }^{14} \mathrm{~N}$ itself is a strong $n$-poison, via the reaction ${ }^{14} \mathrm{~N}(n, p)^{14} \mathrm{C}$.

As for the astrophysical sites for s-processing, a long series of researches have been addressed to the analysis of the He-burning phases in stars of different masses. In massive stars, $\left(M \gtrsim 15 M_{\odot}\right)$, the ${ }^{22} \mathrm{Ne}$ source can only be activated near core He-exhaustion or in the subsequent He-shell phase. The nucleosynthesis of $s$-nuclei has been followed within the framework of different stellar evolutionary scenarios and nuclear reaction networks, with substantial agreement in the results. Since one is faced with a single neutron irradiation, with a fairly low number of neutrons captured per iron seed, significant overproductions are obtained only for $s$-isotopes with $A<80$ (Peters, 1968; Couch et al., 1974; Lamb et al., 1977). Recent revisions in the nuclear reaction rates, particularly the increased rate of the ${ }^{12} \mathrm{C}(\alpha, \gamma){ }^{16} \mathrm{O}$ reaction, and the increased importance of the major light $n$ poisons ${ }^{22} \mathrm{Ne}$ and ${ }^{25} \mathrm{Mg}$, reduce the efficiency of s-processing in He-burning cores (Busso and Gallino, 1985; Thielemann and Arnett, 1985; Gallino and Busso, 1985; Prantzos et al., 1987). Nevertheless, it is still commonly believed that the weak $s$-component has to be ascribed to massive stars so that they can account for the observed s-enhancements in halo Pop. II stars (Spite and Spite, 1978; Barbuy et al., 1985; Krishnaswamy-Gilroy et al., 1988).

In stars less massive than about $8 M_{\odot}$ the He-shell burning phases are potentially more attractive for the synthesis of the bulk of s-elements. These stars, while ascending the asymptotic red giant branch (AGB), suffer a doubleshell burning phase, which acts through recurring thermal instabilities. During a thermal pulse, a convective shell grows in the region between the carbon-oxygen core and the hydrogen-helium discontinuity. This behaviour was discovered by Schwarzschild and Härm (1965) for a $1 M_{\odot}$ star and confirmed for stars in a large 
mass range up to about $8 M_{\odot}$ (Weigert, 1966; Iben, 1975a; Sugimoto and Nomoto, 1975; Becker, 1981 and references therein). Schwarzschild and Härm (1967) found that a small amount of proton-rich material can be dredged down from the external envelope into the convective He shell, thus activating the ${ }^{13} \mathrm{C}$ source. The corresponding s-processing was investigated by Sanders (1967). In a similar context, Ulrich (1973) demonstrated that, independently of the neutron source, an exponential distribution of neutron exposures is naturally achieved by a pulsed mechanism in which the s-processed material undergoes repeated n-irradiations, each followed by a mixture of fresh Fe-seeds and fresh $n$-producing nuclei. Actually, Iben (1976) pointed out that an entropy barrier driven by radiation pressure prevents the penetration of the convective He-shell into the hydrogen-rich envelope, and consequently the ${ }^{13} \mathrm{C}$ source would never operate. Nevertheless, following Iben (1975a,b), in intermediate mass stars (IMS: $3 M_{\odot} \lesssim M \lesssim 8 M_{\odot}$ ), during AGB phases the temperature at the bottom of the convective shell increases from one pulse to another up to a rather high asymptotic value: $T_{b} \gtrsim 3.510^{8} \mathrm{~K}$. In this scenario, the repeated process of n-exposures envisaged by Ulrich would indeed occur in IMS, with the ${ }^{22} \mathrm{Ne}$ source replacing the ${ }^{13} \mathrm{C}$ one. On the basis of this hypothesis, Truran and Iben (1977) showed that in IMS an asymptotic distribution of $s$-process abundances is obtained that fits well the solar abundance pattern for $80 \lesssim A \lesssim 200$.

Since then, many authors (see Mathews and Ward, 1985 and references therein) have examined the details of the $s$-process chain, emphasizing the importance of taking into account several branches. Besides, more detailed calculations on s-processing were made possible by the strong improvements both in the available experimental set of neutron-capture cross sections at stellar energies and in the evaluation of the solar-system abundances. The combined information coming from network calculations in stellar models and from analyses of the s-process soon showed that the ${ }^{22} \mathrm{Ne}$ source suffers serious problems. Indeed, according to Iben (1977), the maximum temperature reached at the bottom of the convective shell increases from $T_{b}=264$ to 415 million degrees for $\mathrm{C}-\mathrm{O}$ core masses ranging from 0.8 to $1.36 M_{\odot}$; consequently, the maximum neutron density varies from $10^{8}$ to $10^{11} \mathrm{~cm}^{-3}$. In these conditions, only for core masses near the minimum limit for the activation of the ${ }^{22} \mathrm{Ne}$ source could the neutron density be reconciled with the $s$-process conditions, but in that case the neutron exposure parameter $\tau_{0}$ is too low. For higher core masses, the high n-densities imply a neutron irradiation intermediate between the $s$ - and the r-process (Despain, 1980), and that would lead to a non-solar distribution of $s$-isotopes. The possibility was envisaged that the final decline of the $n$-density could help in overcoming this difficulty, since the resulting abundances are strongly influenced by the n-density tail (Cosner et al., 1980). However, as pointed out by Howard et al. (1986), the final drop in neutron density is too rapid for stars with high core masses. Another problem arises from the increased effect of the light $n$-poisons, particularly ${ }^{22} \mathrm{Ne}$. Indeed, when adopting the set of cross-sections recommended by Bao and Käppeler (1987: hereafter BK87), the ${ }^{22} \mathrm{Ne}$ source in IMS becomes inefficient in producing the heavy $s$-isotopes (Busso et al., 1988a). The very recent reduction in the cross sections of Ne isotopes suggested by Winters and Macklin (1988) could lead to a possible revision of this scenario, but the achievement of solar isotopic ratios would still be 
precluded by the above considerations. Besides these intrinsic nuclear problems, the activation of the ${ }^{22} \mathrm{Ne}$ source in thermally pulsing IMS is also faced with increasing observational difficulties. Indeed, the number of AGB stars with $M_{b o l}<$ -6 , at which the ${ }^{22} \mathrm{Ne}$ source is supposed to be effective, have not been found in the expected frequency in LMC surveys (Blanco et al., 1980; Cohen et al., 1981; Mould and Reid, 1987; see also Habing, 1987 for a similar problem in the Galaxy). Moreover, together with s-isotopes, the ${ }^{22} \mathrm{Ne}$ source produces high overabundances of ${ }^{25,26} \mathrm{Mg}$, but Smith and Lambert $(1986,1988)$ did not observe in S stars any significant overabundance of these $n$-rich $\mathrm{Mg}$ isotopes relative to ${ }^{24} \mathrm{Mg}$. These facts work against the operation of ${ }^{22} \mathrm{Ne}$ source in IMS. Finally, the observation in low mass AGB stars of the long-lived unstable isotopes ${ }^{99} \mathrm{Tc}$ (Smith and Wallerstein, 1983; Dominy and Wallerstein, 1986; Little et al., 1987, Wallerstein and Dominy, 1988 ), and ${ }^{93} \mathrm{Zr}$ (Peery and Beebe, 1970; Zook, 1978, 1985), clearly indicates that $s$-processing is operating in situ during these phases by a source which has to be different from the ${ }^{22} \mathrm{Ne}$ one. A thorough discussion of the above observational constraints and of their implications for stellar models is given in Hollowell and Iben (1988a).

\section{THE ${ }^{13} \mathrm{C}$ SOURCE}

The problems encountered by the ${ }^{22} \mathrm{Ne}$ source in the synthesis of the main $s$ component in IMS brought many authors to reanalyze the conditions for the activation of the alternative source ${ }^{13} \mathrm{C}(\alpha, n){ }^{16} \mathrm{O}$ in low mass stars (LMS). As previously mentioned, the main difficulty concerning this reaction is that it requires some mixing between the p-rich and the C-rich zones. Iben and Renzini (1982a; 1982b, hereafter IR82) suggested that a suitable mechanism for this process to occur can indeed operate in LMS of low metallicity. After the occurrence of each thermal instability, in the interpulse phase the C-rich material is pushed to low temperatures and the local opacity strongly increases owing to partial recombination of carbon (Sackmann, 1980). This fact allows the formation of a semiconvective zone where a small amount of hydrogen is dredged down into carbon enriched zones. The entropy barrier problem discussed above is thus overcome. About $210^{4} \mathrm{yr}$ later the region heats up and the reaction chain ${ }^{12} \mathrm{C}(p, \gamma){ }^{13} \mathrm{~N}\left(\beta^{+} \nu\right){ }^{13} \mathrm{C}(p, \gamma){ }^{14} \mathrm{~N}$ consumes all the hydrogen, producing a ${ }^{13} \mathrm{C}$ - (and ${ }^{14} \mathrm{~N}$ )-pocket of a few $10^{-4} M_{\odot}$. During the next pulse, this pocket is engulfed in the convective He-shell, where ${ }^{13} \mathrm{C}$ nuclei easily suffer $\alpha$-captures and release neutrons. The effectiveness of this mechanism critically depends on the complex physics of convective boundaries and on the treatment of ${ }^{12} \mathrm{C}$ opacities in the recombination phase. Actually, Boothroyd and Sackmann (1988a) did not find this process to be important in their LMS models; nevertheless, Hollowell and Iben $(1988 \mathrm{~b}, \mathrm{c})$ confirmed the possibility of formation of a consistent ${ }^{13} \mathrm{C}$-pocket. Again, first estimates of neutron densities were in the range of $10^{11}$ to $10^{12} \mathrm{~cm}^{-3}$ (IR82; Malaney, 1986a,b, 1987; Picchio et al., 1988a), thus implying that the ${ }^{13} \mathrm{C}$ source would produce an $s$-isotope distribution far from the solar one. In particular, Malaney (1986c, 1987) pointed out that a too high overabundance of ${ }^{96} \mathrm{Zr}$ would result with respect to ${ }^{90} \mathrm{Zr}$. This fact is at odds with the observations of S stars, showing little or no ${ }^{96} \mathrm{Zr}$ (Peery and Beebe, 1970; Zook, 1978). However, from other points of view the reaction ${ }^{13} \mathrm{C}(\alpha, n){ }^{16} \mathrm{O}$ 


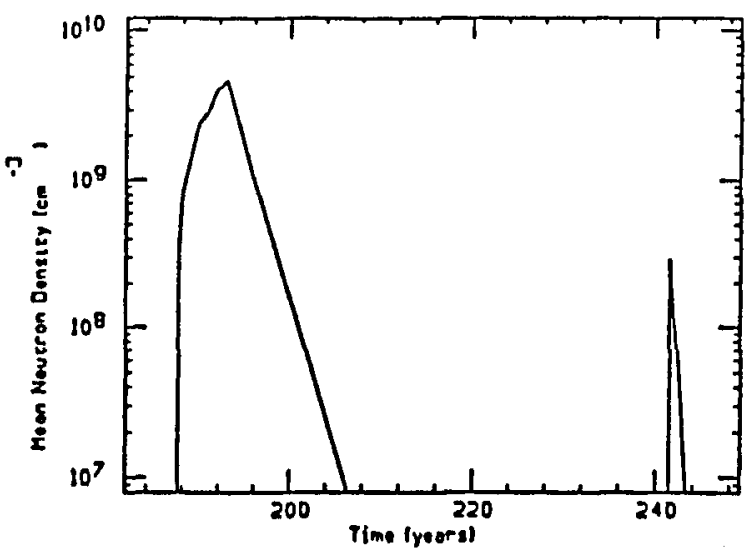

Fig. 2 - Temporal evolution of the neutron density in a low mass AGB star. Time is calculated from the beginning of the thermal pulse. The first neutron burst is caused by the reaction ${ }^{13} \mathrm{C}(\alpha, n){ }^{16} \mathrm{O}$ operating at $T_{b} \simeq 1.518^{8} \mathrm{~K}$, the second neutron burst is caused by the reaction ${ }^{22} \mathrm{Ne}(\alpha, n)^{25} \mathrm{Mg}$ operating at $\mathrm{T}_{b} \simeq 3.010^{8} \mathrm{~K}$.

Table 2

Relative importance of various n-absorbers

a) Cross sections from BK87

\begin{tabular}{|c|c|c|c|c|c|c|}
\hline & ${ }^{12} \mathrm{C}$ & ${ }^{14} N$ & ${ }^{20} \mathrm{Ne}$ & ${ }^{22} \mathrm{Ne}$ & ${ }^{25} \mathrm{Mg}$ & ${ }^{66} \mathrm{Fe}$ \\
\hline$\sigma_{i}(30 \mathrm{keV})$ & 0.2 & 1.7 & 1.1 & 0.9 & 6.5 & 13.1 \\
\hline $10^{4} \sigma_{i} X_{i} / A_{i}$ & 50 & 0.9 & 0.05 & 0.4 & 0.09 & 0.04 \\
\hline \multicolumn{7}{|c|}{ b) New cross sections } \\
\hline & ${ }^{12} \mathrm{C}$ & ${ }^{14} N$ & ${ }^{20} \mathrm{Ne}$ & ${ }^{22} \mathrm{Ne}$ & ${ }^{25} \mathrm{Mg}$ & ${ }^{56} \mathrm{Fe}$ \\
\hline$\sigma_{i}(30 \mathrm{keV})$ & 0.2 & 0.81 & 0.12 & $0.045-0.24$ & 6.5 & 13.1 \\
\hline $10^{4} \sigma_{i} X_{i} / A_{i}$ & 50 & 0.4 & 0.04 & $0.02-0.10$ & 0.09 & 0.04 \\
\hline
\end{tabular}


would offer some important advantages. Indeed: (i) it would not imply a too large production of $n$-rich $\mathrm{Mg}$ isotopes; (ii) it would be consistent with the observation of ${ }^{99} \mathrm{Tc}$ (and ${ }^{93} \mathrm{Zr}$ ) in LMS of $M_{b o l}$ as low as -4.0 (corresponding to a core mass $M_{\mathrm{c}} \simeq 0.6 M_{\odot}$ ).

In this framework, following the IR82 suggestion for the formation of the ${ }^{13} \mathrm{C}$-pocket, the pulse model by Iben (1982) for a $0.7 M_{\odot}, Z=0.001$ star has been reanalyzed by Gallino et al. (1988a), incorporating a large network of reactions that includes charged-particle processes from $\mathrm{He}$ to $\mathrm{Si}$ and $n$-captures from $\mathrm{C}$ to Po (see Picchio et al., 1988b for details). The network takes into account more than 100 branching points along the 8 -chain as well as the reaction channels of the most important isomeric states and adopts the recommended neutron cross sections by BK87, further improved by Ratynski and Käppeler (1988) and Beer and Macklin (1988b). The set of $\beta$-decay lifetimes at stellar conditions is from Takahashi and Yokoi (1987), Fuller et al. (1983) and Klay and Käppeler (1988). The adopted solar-system abundances are from Anders and Ebihara (1982). The process of engulfment of the ${ }^{13} \mathrm{C}$-pocket formed during the interpulse period has been followed in detail, assuming the $11^{\text {th }}$ pulse by Iben (1982) as representative of the asymptotic thermal instabilities. The duration of the entire ingestion phase is of about $5.5 \mathrm{yr}$; the rate of ingestion $\left(710^{-5} M_{\odot} / y r\right)$ corresponds to the growing rate of the external profile of the convection zone and was assumed to be constant. As long as ${ }^{13} \mathrm{C}$ is ingested, it is mixed over the convective region and suffers $\alpha$-captures. Next, the surviving ${ }^{13} \mathrm{C}$ is brought to exhaustion as He-burning proceeds. The ${ }^{13} \mathrm{C}(\alpha, n){ }^{16} \mathrm{O}$ reaction takes place at a mild temperature: $T_{b} \simeq 1.510^{8}$ $K$. The entire neutron irradiation lasts for about 30 years, reaching an exposure $\Delta \tau\left({ }^{13} \mathrm{C}\right) \simeq 0.11 \mathrm{mb}^{-1}$.

The resulting mean neutron density $\bar{N}_{n}$ in the shell is far from being constant (contrary to what is currently assumed in the classical analysis of the sprocess), reaching a peak of about $310^{9} \mathrm{~cm}^{-3}$ at the end of the ingestion phase and then decreasing smoothly (Fig. 2). The strong reduction of the neutron density with respect to the previous estimates is obtained thanks to the damping effect of three moderating phenomena: (i) the temperature and density stratification of the convective zone, (ii) the realistic (low) ingestion rate of ${ }^{13} \mathrm{C}$, (iii) the neutron recycling introduced by $n$-captures on ${ }^{12} \mathrm{C}$. Concerning this last effect, one has to notice that ${ }^{12} \mathrm{C}$ nuclei are very abundant in the He-shell, so that a very small $n$-capture cross section would be sufficient to let them become the major neutron poisons. The neutrons captured by ${ }^{12} \mathrm{C}$ are not lost, but recycled through the sequence ${ }^{12} \mathrm{C}(\mathrm{n}, \gamma){ }^{13} \mathrm{C}(\alpha, n){ }^{16} \mathrm{O}$. The importance of this $n$-recycling by ${ }^{12} \mathrm{C}$ for reproducing a solar-system distribution of $s$-isotopes has to be underlined. This chain acts as a reservoir for neutrons, allowing them to be released at delayed times, the peak $\bar{N}_{n}$ to be reduced and the $\bar{N}_{n}$ tail to decline smoothly. Furthermore, the $n$-recycling on ${ }^{12} \mathrm{C}$ has the effect of reducing the consequences of the somewhat uncertain ingestion rate. Actually, the $n$-capture cross section of ${ }^{12} \mathrm{C}$ at astrophysical energies is poorly known: the theoretical evaluation by Fowler et al. (1967) at $30 \mathrm{keV}$ is as low as $0.003 \mathrm{mb}$, while the experimental value by Allen et al. (1971) is higher, $\sigma_{12}=(0.2 \pm 0.4) \mathrm{mb}$, though affected by a large uncertainty. A recent theoretical 
estimate (Reffo, 1988) gives $\sigma_{12}=(0.02 \pm 0.01) \mathrm{mb}$. The relative importance of $n$-captures on ${ }^{12} \mathrm{C}$ with respect to the other major light poisons can be evaluated by comparing the various products $N_{i}\langle\sigma v\rangle_{i}$ or, apart from a common factor, the products $\left(X_{i} / A_{i}\right) \sigma_{i}$, which are shown in Table 2 . Typical abundances by mass $X_{i}$ at the $\bar{N}_{n}$ peak have been used. From Table 2a it appears that ${ }^{12} \mathrm{C}$ dominates over all other $n$-poisons whenever $\sigma\left({ }^{12} \mathrm{C}\right) \geq 0.006 \mathrm{mb}$. The effect is even greater when adopting the most recent estimates for the $n$-capture cross sections on ${ }^{14} \mathrm{~N}$ (Brehm et al., 1988) and on Ne isotopes (Winters and Macklin, 1988), as shown in Table 2b. In this case ${ }^{12} \mathrm{C}$ dominates whenever $\sigma\left({ }^{12} \mathrm{C}\right) \geq 0.002 \mathrm{mb}$. Also $n$-captures on ${ }^{14} \mathrm{~N}$ could give rise to a $n$-recycling effect (Jorissen and Arnould, 1986; Brehm et al., 1988), through the reaction ${ }^{14} \mathrm{~N}(n, p){ }^{14} \mathrm{C}$, followed by ${ }^{12} \mathrm{C}(p, \gamma){ }^{13} \mathrm{~N}\left(\beta^{+} \nu\right){ }^{13} \mathrm{C}(\alpha$, $n)^{16} \mathrm{O}$. Nevertheless, during the first ingestion phase, that lasts for about 3.4 years and accounts for about half of the ${ }^{13} \mathrm{C}$ dredged-down, the abundance of ${ }^{14} \mathrm{~N}$ is low; in the second phase of ingestion, where ${ }^{14} \mathrm{~N}$ is an important $n$-poison, its recycling accounts for less than $15 \%$ of the neutrons released, owing to the concurrent $n$ poisoning by ${ }^{22} \mathrm{Ne}$ and to the fact that more than about $50 \%$ of protons get lost via other $p$-channels, mainly ${ }^{18} \mathrm{O}(p, \alpha){ }^{15} \mathrm{~N}$.

Near the end of the pulse, the convective shell grows rapidly to its maximum extension, and the s-processed zone is remixed with $40 \%$ of He-rich matter containing fresh ${ }^{14} \mathrm{~N}$ and fresh iron seeds. There, the temperature at the bottom of the shell sharply increases up to $T_{b}=310^{8} \mathrm{~K}$ and then decreases exponentially. All the ${ }^{14} \mathrm{~N}$ nuclei are transformed into ${ }^{22} \mathrm{Ne}$ via the chain ${ }^{14} \mathrm{~N}(\alpha, \gamma){ }^{18} \mathrm{~F}\left(\beta^{+} \nu\right){ }^{18} \mathrm{O}(\alpha, \gamma){ }^{22} \mathrm{Ne}$, about $1 \%$ of ${ }^{22} \mathrm{Ne}$ further suffering $\alpha$-captures. Consequently, a second burst of neutrons at high temperature is released by the reaction ${ }^{22} \mathrm{Ne}(\alpha, n)^{25} \mathrm{Mg}$, which lasts for about 2 years, $\bar{N}_{n}$ reaching a maximum value of about $410^{8} \mathrm{~cm}^{-3}$. The total neutron exposure of the pulse is not significantly affected, being $\Delta \tau\left({ }^{22} \mathrm{Ne}\right) \simeq 110^{-3} \mathrm{mb}^{-1}$. Subsequently, in the interpulse period, which in Iben's $0.7 M_{\odot}$ model lasts for about $210^{5} \mathrm{yr}$, the long-lived nuclei produced during the two irradiations are allowed to decay according to their lifetimes. The process of nucleosynthesis was followed for 20 subsequent pulses. Typically, an asymptotic distribution of $s$-isotopes is reached after about 10 pulses.

\section{ANALYSIS OF $s$-PROCESS CONTRIBUTIONS}

The behaviour of the $n$-density sketched in Fig. 2, and in particular its long tail, is exactly what one needs for producing a solar $s$-isotope distribution, as stressed by Howard et al. (1986). Indeed, the resulting overabundances of s-only and prevailing- $s$ nuclei for $A>80$ are remarkably flat (Fig. 3), with a strong overproduction factor of about 2000. The various isotopes shown in Fig. 3 have been indicated with different symbols according to the analysis of Käppeler, Beer and Wisshak (1988) of their relative s-contributions. Overall, the agreement is very stringent; the only exceptions are the so-called $r$-only isotopes and the two isotopes ${ }^{86} \mathrm{Kr},{ }^{87} \mathrm{Rb}$. Concerning the former ones, the choice of "a priori" $r$-only isotopes might be uncorrect, since these nuclei are not shielded by the s-process chain. In Fig. 3 no correction has been applied for the very long-lived nuclei with $\tau>10^{10} \mathrm{yr}$ and their by-products. When this correction is introduced, 
Case IR82, $\sigma(\mathrm{C} 12)=0.1, \sigma(\mathrm{N} 14)=1.7, \sigma(\mathrm{NE} 20)=1.4, \sigma(\mathrm{NE2} 2)=0.9$

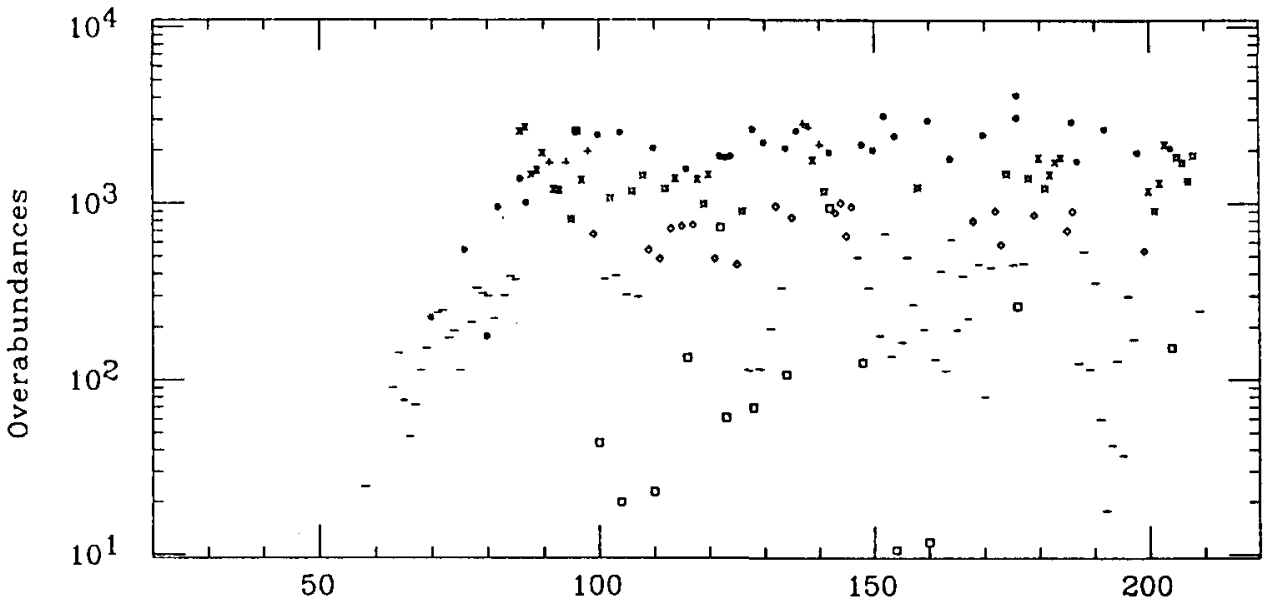

Atomic mass number

Fig. 3. Overabundances of heavy isotopes in a low mass star following the 1882 model. The n-capture cross sections at $30 \mathrm{keV}$ for the major light $n$-poisons are indicated at the top (in mb). The 8 -only isotopes are denoted by $a *$. The prevailing-s isotopes are denoted by $a+(80 \leq s$-MAIN $<100)$, or by a $\times(60 \leq 8$-MAIN $<80)$, where $s-$ MAIN is the relative contribution for the main s-component, according to the analysis of Kappeler, Beer and Wisshak (1988). Isotopes with minor $s$-contributions are denoted by a starburst $(40 \leq 8$-MAIN $<60)$, by a $\circ(20 \leq s-\mathrm{MAIN}<40)$, or by a - $(0 \leq 8-\mathrm{MA} I N<20)$. The "r-only" isotopes are denoted by an open square. Initial abundances are Irom Anders and Ebihara (1982). A quite similar distribution is obtained by reducing the $n$-cross section on ${ }^{12} \mathrm{C}$ down to $\sigma \simeq 0.003 \mathrm{mb}$.

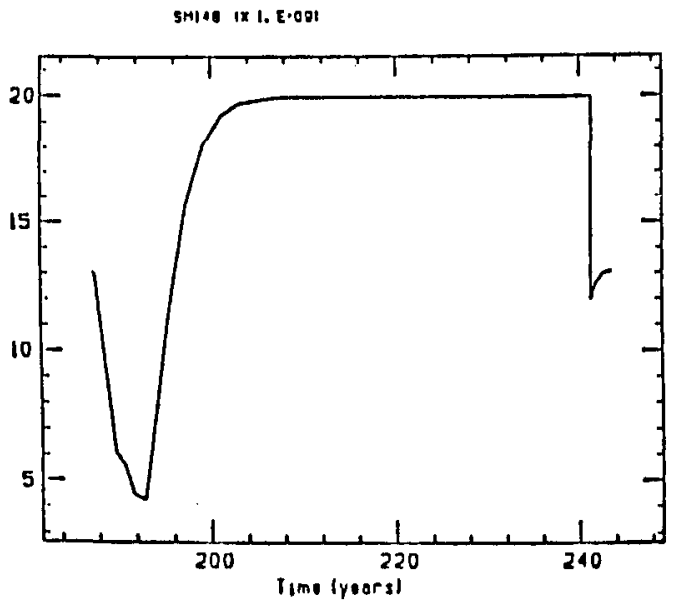

Fig. 4 - Temporal evolution of the mass abundance of ${ }^{148} \mathrm{Sm}$, a typical branching-dependent s-only isotope. The initial decrease is an effect of the high peak $\bar{N}_{n}$. The mass abundance is frozen out near ${ }^{13}$ C-exhaustion when $\bar{N}_{n} \simeq 210^{8}$ $\mathrm{cm}^{-3}$. At $t=241$ y the convective shell rapidly expands up to its maximum extent, dredging down $40 \%$ of the He-rich matter containing original seeds. At the right the small eflect of the high temperature phase is shown. 
a substantial improvement is obtained for ${ }^{87} \mathrm{Sr},{ }^{176} \mathrm{Lu}$ and ${ }^{187} \mathrm{Os}$ (Busso et al., 1988b).

Typical behaviours in time of the abundances by mass of s-only isotopes are shown in Fig. 4 and 5. By comparison with Fig. 2, showing the temporal variation of $\bar{N}_{n}$, one can notice that the abundances of most s-isotopes are frozen out when $\bar{N}_{n} \lesssim 510^{8} \mathrm{~cm}^{-3}$. The small contribution by the ${ }^{22} \mathrm{Ne}$ source working at higher temperature is shown at the right. There are a few s-only isotopes that are destroyed by the neutron irradiation of the ${ }^{13} \mathrm{C}$ source at mild temperature, and are instead produced by the activation of the ${ }^{22} \mathrm{Ne}$ source (Fig. 6). They are: ${ }^{64} \mathrm{Zn},{ }^{80} \mathrm{Kr},{ }^{152} \mathrm{Gd}$, and ${ }^{164} \mathrm{Er}$. These isotopes are $\beta$-decay products of branchings at ${ }^{63} \mathrm{Ni},{ }^{79} \mathrm{Se},{ }^{151} \mathrm{Sm}$, and ${ }^{163} \mathrm{Dy}$ that are strongly temperature dependent. One has to notice that the long-lived isotopes ${ }^{99} \mathrm{Tc}$ and ${ }^{93} \mathrm{Zr}$ are produced in noticeable amounts during ${ }^{13} \mathrm{C}$ consumption and survive the short high temperature phase, in agreement with observational evidences. Other similar interesting cases are provided by ${ }^{107} \mathrm{Pd},{ }^{135} \mathrm{Cs}$, and ${ }^{210} \mathrm{Bi}$.

Two crucial discrepancies between observations and theoretical expectations, working against the reliability of the ${ }^{13} \mathrm{C}$ source in LMS, are now significantly alleviated. The first one concerns the ratio ${ }^{96} \mathrm{Zr} /{ }^{90} \mathrm{Zr}$. The computations presented here reduce this ratio by a factor of 7 with respect to Malaney (1987), though from Fig. 3 one sees that ${ }^{96} \mathrm{Zr}$, currently considered an r-only isotope, has an overproduction factor of the same order of the prevailing-s ${ }^{90} \mathrm{Zr}$. Nevertheless, since the solar relative abundance of ${ }^{86} \mathrm{Zr}$ is as low as $2.78 \%$, at this level it would be hardly detectable in Ba stars. The second major problem is the too high predicted ratio of $\mathrm{Rb} / \mathrm{Sr}$ (Malaney, 1987). Again, a consistent reduction by a factor of 3 is now obtained, though this is not yet enough if one wants to match observations of $\mathrm{Ba}$ stars, such as $\zeta$ Cap, or HR774 (see Malaney and Lambert, 1988). A possible solution consists in reducing the duration of the pulsed neutron exposure, as suggested by Beer and Macklin (1988b), but further stellar evolutionary calculations for different core mass stars are needed to check this point.

\section{COMPARISON WITH THE CLASSICAL s-PROCESS ANALYSIS}

The classical analysis assumes a steady flow at constant $N_{n}$ with constant temperature and matter density. Following the discussion of Käppeler (1986), the neutron density of the main s-component can then be determined from the analysis of particular branchings that are not temperature dependent. The branching at ${ }^{185} \mathrm{~W}$, that is responsible for the formation of the s-only isotope ${ }^{186} \mathrm{Os}$, gives the best indication: $N_{n}=(1.3 \pm 0.7) 10^{8} \mathrm{~cm}^{-3}$ (Beer and Macklin, 1988a). Similar indications are provided by the branchings at ${ }^{147,148} \mathrm{Pm}$ and ${ }^{169} \mathrm{Er}$. Once an estimate of $N_{n}$ has been obtained, the temperature can be evaluated from branchings that are strongly temperature dependent. This occurs in particular at ${ }^{151} \mathrm{Sm}$, from which one deduces a temperature ranging between 210 and 350 million degrees (Beer and Macklin, 1988a). Furthermore, the analysis of the ${ }^{163} \mathrm{Dy}-{ }^{163} \mathrm{Ho}$ branching, in which bound state $\beta$-decays are activated at high temperature, allows an evaluation of the electron density: $n_{e} \simeq(0.8 \div 4) 10^{27} \mathrm{~cm}^{-3}$, corresponding to a matter 


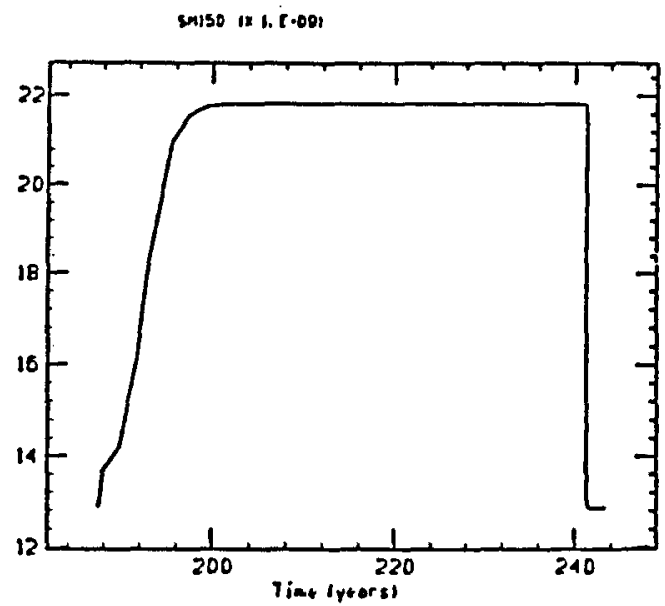

Fig. 5 - Temporal evolution of the mass abundance of ${ }^{150} \mathrm{Sm}$, a typical sonly isotope dependent on the main s-fluence.

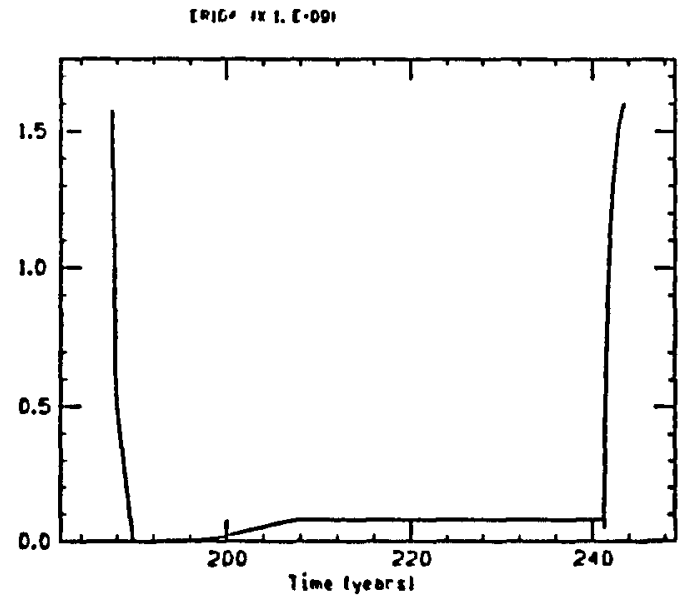

Fig. 6 - Temporal evolution of the mass abundance of ${ }^{164} \mathrm{Er}$, a typical high-temperature dependent s-only isotope. This isotope is destroyed by the n-burst operating at low temperature, whereas it is synthesized during the short high temperature $n$-burst of the ${ }^{22} \mathrm{Ne}$ source. Other isotopes showing the same behaviour are ${ }^{64} \mathrm{Zn},{ }^{80} \mathrm{KI}$ and ${ }^{152} \mathrm{Gd}$. 
density: $\rho \simeq(2.6 \div 13) 10^{3} \mathrm{~g} \mathrm{~cm}^{-3}$ (Beer, Walter and Macklin, 1985). In this respect, ${ }^{163} \mathrm{Dy}$ acts as a barometer. Finally, it is possible to obtain evidence of the pulsed nature of the main s-component and of the duration of each exposure. Indeed, following Ward and Newman (1978), Beer (1986b), and Beer and Macklin $(1988 \mathrm{a}, \mathrm{b})$, the difference between a continuous s-process and the case of repeated pulses becomes evident for those branchings for which the time duration $\Delta t$ of the neutron exposure is of the order of the total lifetime of the unstable isotope $t_{\text {tot }}=$ $\left(\lambda_{\beta}+\lambda_{n}\right)^{-1}$ (where $\lambda_{\beta}$ is its $\beta$-decay rate and $\lambda_{n}=N_{n}\langle\sigma v\rangle_{n, \gamma}$ its $n$-capture rate, respectively). The only interesting branchings are those at ${ }^{85} \mathrm{Kr}$ and at ${ }^{151} \mathrm{Sm}$. The pulsed nature of the main component appears to be clearly established; from the analysis of the first branching the pulse duration $\Delta t$ must not exceed about 30 years, while from the second one a lower limit of about 3 years is deduced (Beer and Macklin, 1988b).

When comparing all the above prescriptions with the results of the LMS pulsed model (Busso et al., 1988b), one has to take into account the situation concerning the neutron exposure (see Fig. 2). It can be summarized in three steps: (i) mild temperature and high $\bar{N}_{n}$ phase; (ii) mild temperature phase with exponentially decreasing $\bar{N}_{n}$; (iii) high temperature phase and low $\bar{N}_{n}$ burst. Considering the s-only isotopes that in the classical analysis allow a determination of neutron density, one sees that ${ }^{148} \mathrm{Sm},{ }^{170} \mathrm{Yb}$, and ${ }^{186} \mathrm{Os}$ are first destroyed at high $\bar{N}_{n}$ and then produced during the $\bar{N}_{n}$ tail, their abundance being frozen out when typically $\bar{N}_{n}=(1-2) 10^{8} \mathrm{~cm}^{-3}$, as shown by Fig. 4 . Consequently, the two indications of $\bar{N}_{n}$ are in a fine agreement. All other isotopes lying on the main s-process path, e.g. ${ }^{150} \mathrm{Sm}$ (see. Fig. 5), are essentially dependent on the integral exposure $\tau$, and not on the specific behaviour of the neutron density.

As for temperature, we have already stated that the ${ }^{13} \mathrm{C}$ source operates at lower temperatures with respect to those indicated by the classical analysis, that are better indicative of the ${ }^{22} \mathrm{Ne}$ source. Nevertheless, in LMS, while the bulk of the $s$-isotopes is synthesized at a mild temperature, it is just the marginal activation at high temperature of the ${ }^{22} \mathrm{Ne}$ source that leads the thermometers ${ }^{152} \mathrm{Gd},{ }^{164} \mathrm{Er}$ (and ${ }^{80} \mathrm{Kr}$ ) to be produced at the expected ratios.

Concerning further the information on density, when the convective shell expands to its full extent and ${ }^{163} \mathrm{Dy}$ decays at high temperature, the average density is about $510^{3} \mathrm{~g} \mathrm{~cm}^{-3}$, in agreement with the prescriptions quoted above. Finally, also the duration of the pulse is not inconsistent with the above estimates, being about 30 years for the main $n$-burst (which is responsible for the production of ${ }^{86} \mathrm{Kr}$ and ${ }^{87} \mathrm{Rb}$ ), and about 2 years for the high temperature one (which is responsible for the production of ${ }^{162} \mathrm{Gd}$ ). Nevertheless, in this case the real pulse conditions are not strictly comparable with the classical analysis, owing to the different temperature and $\bar{N}_{n}$ history. Anyway, the overproduction of ${ }^{152} \mathrm{Gd}$ resulting from the LMS model is quite satisfactory, as shown in Fig. 3. Also the production of ${ }^{86} \mathrm{Kr}$ and ${ }^{87} \mathrm{Rb}$ appears typical of s-only isotopes, though their interstellar abundances could also receive a further contribution from the weak 
component, as well as from the r-process.

The main asymptotic characteristics of the pulsed s-process are illustrated in Table 3, first column. From the analysis of Ulrich (1973), the repeated pulse mechanism implies that the number $n_{c}$ of neutrons captured per iron seed (i.e. $\left.\Sigma_{i}\left(A_{i}-56\right) N_{i} / N_{56}\right)$ and the neutron exposure parameter $\tau_{0}$ are given by the formulae: $n_{c}=\Delta n_{c} /(1-r)$ and $\tau_{0}=-\Delta \tau / \ln r$, where $r$ is the overlapping factor between two subsequent pulses, and $\Delta n_{c}, \Delta \tau$ are the corresponding parameters for each pulse. However, as for the evaluation of $n_{c}$, one has to realize that the pulsed process in LMS is different from the simplified assumption by Ulrich, since now the fresh $\mathrm{Fe}$ is ingested near the end of each pulse, and only partially burnt, whereas in the Ulrich's case no fresh Fe is left at the end of the pulse. Consequently, the first relation has to be replaced by: $n_{c}=\Delta n_{c} r /(1-r)$. With $r=0.6$ in the IR82 case, one has: $n_{c}=7.50 \times 0.6 /(1-0.6)=11.25$ and $\tau_{0}=-0.104 / \ln 0.6=0.20$. The value of $n_{\mathrm{c}}$ is in very good agreement with the result by the classical s-analysis (see Table 1). As for the exposure parameter $\tau_{0}$, the best-fit value deduced from the analysis of the $\sigma N_{s}$ distribution, $\tau_{0}=0.30\left[(\mathrm{kT} /(30 \mathrm{keV})]^{1 / 2} \mathrm{mb}^{-1}\right.$, corresponds to $\tau_{0} \simeq 0.18 \mathrm{mb}^{-1}$ for $\mathrm{kT} \simeq 11 \mathrm{keV}$, as in the LMS shell model. Again, the agreement is satisfactory, as yet shown from the flatness of the distribution curve of $s$-overproductions. In summary, one can say that, when properly considered, all the relevant parameters deduced from the classical s-process theory are consistent with the main findings of the pulsed $n$-capture process in LMS.

\section{UNCERTAINTIES IN THE CROSS SECTIONS OF LIGHT $n$ - POISONS AND NEW PULSE MODEL BY HOLLOWELL AND IBEN}

Apart from ${ }^{12} \mathrm{C}$, the uncertainty in the $n$-capture cross sections of the major light $n$ poisons, such as ${ }^{14} \mathrm{~N}$ and ${ }^{22} \mathrm{Ne}$, can modify the overall picture of $s$-nucleosynthesis. Picchio et al. (1988b) have spanned the whole range of uncertainties, assuming the corresponding $n$-capture cross sections as free parameters. As for ${ }^{22} \mathrm{Ne}$, when its cross section is reduced down to $\sigma_{22} \simeq 0.3 \mathrm{mb}$, which is consistent with the most recent estimates by Winters and Macklin (1988), the distribution of $s$-isotopes remains flat, while the average overproduction increases by a factor of about 2; for lower values there is a tendency to exceedingly produce the heaviest isotopes. Concerning ${ }^{14} \mathrm{~N}$, the reduction by a factor of about 2 of its $n$-capture cross section (Brehm et al., 1988) with respect to the theoretical estimate by Fowler et al. (1967) implies similar considerations, though by a lesser extent.

A more detailed analysis of the possibility of formation of the semiconvective region during the interpulse phase in LMS of low metallicity has been recently made by Hollowell and Iben (1988b). Their new treatment of carbon opacities and of convection confirms the possibility that LMS provide the correct neutron exposures for the synthesis of the main s-component. In their model, the ingestion rate and the overlapping factor are slightly lower than in IR, being now $d M / d t=510^{-5} M_{\odot} / y r$ and $r=0.5$. While in the first phase of ingestion, where ${ }^{13} \mathrm{C} /{ }^{14} \mathrm{~N}>1$, the amounts of ${ }^{13} \mathrm{C}$ and ${ }^{14} \mathrm{~N}$ are about the same in the two cases, the total ${ }^{13} \mathrm{C}$ engulfed is a factor of 2 lower, and the total amount of ${ }^{14} \mathrm{~N}$ is about a factor of 2.5 greater. The distribution of overabundances obtained when recalcu- 
Case 11188, $\sigma(\mathrm{C} 12)=0.1, \sigma(\mathrm{N} 14)=0.81, \sigma(\mathrm{NE20})=0.12, \sigma(\mathrm{NE} 22)=0.24$

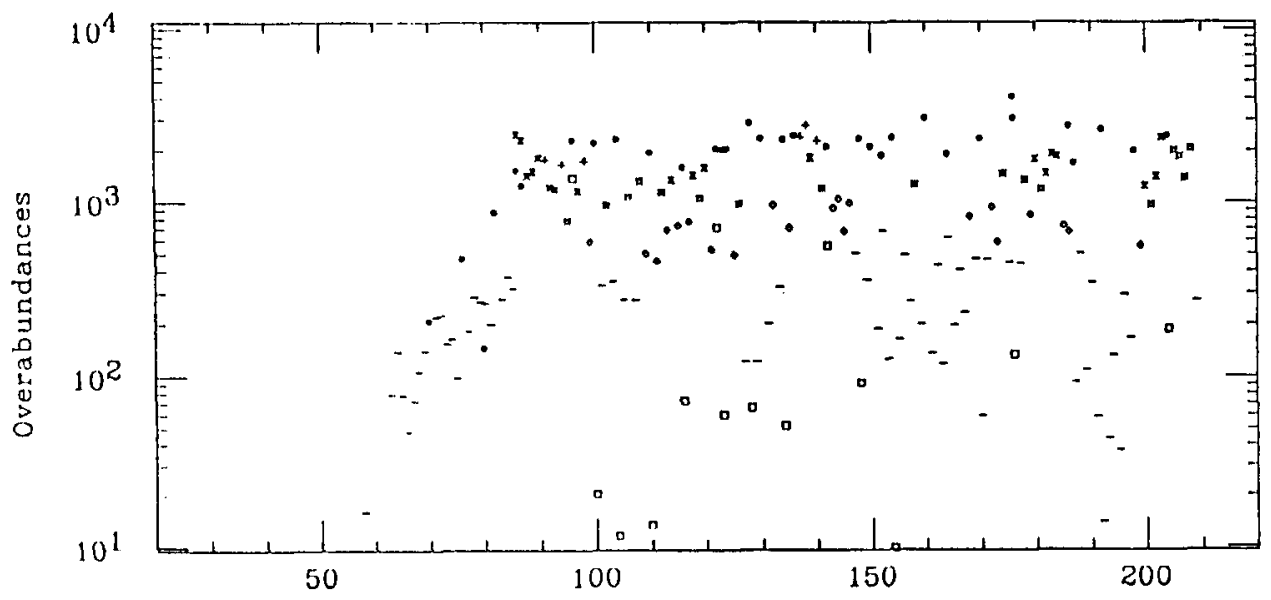

Atomic mass number

Fig. 7. Distribution of the overabundances of heavy isotopes in the model of Hollowell and lben (1988), adopting the reduced ncross sections of light n-poisons shown at the top (in mb). The same distribution is obtained when reducing the n-caplure cross section on ${ }^{12} \mathrm{C}$ down to $\sigma_{13} \simeq 0.003 \mathrm{mb}$. Inilial abundances are from Anders and Ebihara (1982). Notations for the various isotopes are the same as in Fig. 3.

TABLE 3

CIIAILACTERISTICS OF THE NEUTRON IRIADIATION

\begin{tabular}{|c|c|c|}
\hline Parameler & Lit82 & H188 \\
\hline$\Delta n_{c}$ & 7.89 & 11.30 \\
\hline$\left(\kappa_{n}\right)_{M}$ & $4.7(09)$ & $3.1(09)$ \\
\hline$\Delta \tau$ & 0.110 & 0.154 \\
\hline$n_{c}$ & 11.83 & 11.30 \\
\hline 10 & 0.215 & 0.222 \\
\hline
\end{tabular}


lating the s-nucleosynthesis with these new prescriptions and adopting the most recent data for the n-capture cross sections of light $n$-poisons is shown in Fig. 7. Despite the differences about the formation and engulfment of the ${ }^{13} \mathrm{C}$-pocket and the reduced effect of light $n$-poisons, the distribution of s-isotopes is hardly distinguishable from that of Fig. 3. However, an improved reduction in the abundances of $r$-only isotopes, such as ${ }^{96} \mathrm{Zr}$, is now obtained.

\section{CONCLUSION}

So far, the possibility of formation of the ${ }^{13} \mathrm{C}$-pocket has been envisaged only for low mass stars of low metallicity. This is presently the main theoretical problem concerning the ${ }^{13} \mathrm{C}$ source. A low metallicity favours the occurrence of semiconvection in the intershell region and makes the thermal instability stronger (Iben, 1983; Boothroyd and Sackmann, 1988b). Consequently, both the third dredge-up and the formation of the ${ }^{13} \mathrm{C}$-pocket are favoured. Stars of $1.5 M_{\odot}$ with metallicity 0.003 and 0.006 have been followed by Lattanzio (1987) up to AGB phases, both showing carbon dredge-up. The MS, S, CS, C, CH and $\mathrm{Ba}$ stars showing $s$-enhancements are typically disc population stars, with metallicities of the order of the solar one or slightly lower (particularly the $\mathrm{CH}$ and $\mathrm{Ba}$ stars, see Cowley and Downs, 1980; Luck and Bond, 1982; Lambert, 1985; Tomkin and Lambert, 1986). In all these peculiar stars the observed ${ }^{12} \mathrm{C}$ is enhanced, thus indicating that the third dredge-up did occur. Then, between the intershell and the envelope a hydrogen/carbon discontinuity forms, which may favour the penetration of a small amount of protons into the carbon-rich zone (Iben and Renzini, 1983). More detailed stellar evolutionary calculations of LMS with different metallicities in AGB phases with improved carbon opacities and treatment of convection are highly desirable. Anyway, to have a first idea of the expectations of the $s$-abundance distribution in stars of different metallicity, one has to outline the importance of the initial abundance of light $n$-poisons. Indeed, while the ${ }^{13} \mathrm{C}$ and ${ }^{14} \mathrm{~N}$ nuclei produced in the semiconvective zone derive from $p$-captures on the newly formed ${ }^{12} \mathrm{C}$ and are independent of the original metal content, the Fe seeds and the light $n$-poisons increase with metallicity. As a first guess, one can assume that the general characteristics of the thermal pulses and of the ${ }^{13} \mathrm{C}$-pocket remain unchanged while varying the metallicity. The results so far obtained (Raiteri et al., 1988) show that, going towards high $Z$ values, the efficiency of the s-processing continuously decreases due to the increased light $n$-poisons. The distribution of overabundances remains flat up to about $Z \simeq Z_{\odot} / 3$, whereas for higher metallicities a sharp discontinuity occurs near the magic-neutron nucleus ${ }^{86} \mathrm{Kr}$. For very metal poor stars the situation is different. Indeed, on one side the decrease of the initial metallicity reduces the amount of $\mathrm{Fe}$ seeds, and this tends to favour the process of $s$-nucleosynthesis; on the other hand, one has to take into account the effect of the primary ${ }^{14} \mathrm{~N}$-nuclei produced together with ${ }^{13} \mathrm{C}$, that do not vary with the metal content. Consequently, again keeping the efficiency of the ${ }^{13} \mathrm{C}$-pocket unchanged, an asymptotic condition is reached for $Z \lesssim 10^{-4}$, with $\Delta \tau \simeq 0.15$ and overproduction ratios increasing from 3500 up to 7000 for the heaviest $s$-only isotopes.

A few noticeable effects concerning isotope ratios are obtained with in- 
creasing metallicity. First of all, the ratio ${ }^{96} \mathrm{Zr} /{ }^{80} \mathrm{Zr}$ decreases by a factor of 3 in disc population stars. This allows for a full explanation of the ${ }^{96} \mathrm{Zr}$ controversy. More interesting, the relative ratios ${ }^{86} \mathrm{Kr} /{ }^{82} \mathrm{Kr}$ and ${ }^{80} \mathrm{Kr} /{ }^{82} \mathrm{Kr}$ decrease with increasing metallicity, whereas ${ }^{83} \mathrm{Kr} /{ }^{82} \mathrm{Kr}$ and ${ }^{84} \mathrm{Kr} /{ }^{82} \mathrm{Kr}$ ratios remain unchanged. This fact may provide a full explanation (Gallino et al., 1988b) of the strange behaviour shown by the exotic $s-\mathrm{Kr}$ inclusions in the Murchison meteorite (Ott et al., 1988; Clayton, 1988; Jorgensen, 1988).

Reproduction of the solar-system distribution of the s-isotopes is the first goal to be achieved. Even if the efficiency of $s$-processing in LMS with increasing metallicity may be questionable, in the framework of current theories of chemical galactic enrichment the main contribution of LMS to the solar s-enrichment has to come from stars of lower metallicity, formed near the end of the galactic halo phase (Busso and Gallino, 1983). For them $\left(Z \simeq 510^{-3}\right)$ a consistent and flat distribution of the heavy $s$-isotopes in the He-shell can still be obtained.

The second main task is an attempt to reproduce the s-enhancements shown by AGB stars. Smith and Lambert $(1985,1986)$, and Smith et al. (1987) showed that the s-process abundance distributions in MS and S stars are described by a large range of neutron exposures: $\tau_{0} \simeq 0.1-0.6 \mathrm{mb}^{-1}$, with an average value well consistent with the classical s-analysis and with the results discussed above. A possible spread of neutron exposures is expected in LMS, when several aspects like the variation of the temporal profile of the convective zone in the first pulses, the temporal variation of the overlapping factor (see Iben, 1982), the amount of the ${ }^{13} \mathrm{C}$ ingested and the metallicity are more carefully considered. For example, the higher s-enhancements shown by Ba stars could be related to their relatively lower metallicity. Furthermore, the MS and S stars could have suffered only a few thermal pulses and the asymptotic conditions for $s$-processing could have not been achieved.

\section{ACKNOWLEDGMENTS}

I deeply acknowledge Maurizio Busso, Franz Käppeler, Guido Picchio, Claudia M. Raiteri, Gianni Reffo and Alvio Renzini, for continuous discussions and collaboration in obtaining the new results presented here. In particular I thank G. Picchio and $M$. Busso for help in the discussion and redaction of the present paper. I thank Hermann Beer for the permission of reproducing Fig. 1. I am very indebted to Icko Iben, Jr. and Dave Hollowell for providing me their new results on the formation of the ${ }^{13} \mathrm{C}$-pocket and for a full and encouraging discussion during this Conference.

\section{REFERENCES}

Allen, B. J., Gibson, J. H., and Macklin, R. L. 1971, Adv. Nucl. Phys., 4, 205. Anders, E., and Ebihara, M. 1982, Geochim. Cosmochim. Acta, 46, 2263. Bao, Z. Y., and Käppeler, F. 1987, Atomic Data and Nucl. Data Tables, 36, 411. 
Barbuy, B., Spite, F., and Spite, M. 1985, Astr. Ap., 144, 343.

Becker, S. A. 1981, in Physical Processes in Red Giants, ed. I. Jr. Iben and A. Renzini (Dordrecht: Reidel), p.141.

Beer, H. 1986a, in Nucleosynthesis and its Implications on Nuclear and Particle Physics, ed. by J. Audouze and N. Mathieu (Dordrecht: Reidel), p. 263.

Beer, H. 1986b, in Advances in Nuclear Astrophysics, ed. E. Vangioni-Flam, J. Audouze, M. Cassé, J. P. Chieze and J. Tran Thanh Van (Paris: Editions Frontières), p. 375.

Beer, H., and Macklin, R. L. 1988a, Ap. J., 331, 1047.

Beer, H., and Macklin, R. L. $1988 \mathrm{~b}$, Measurements of the ${ }^{86,87} \mathrm{Rb}$ Capture Cross Section for s-Process Studies, Ap. J., in press.

Beer, H., Walter, G., and Macklin, R. L. 1985, in Capture Gamma-Rays Spectroscopy and Related Topics, ed. by S. Raman (New York: American Institute of Physics), p. 778.

Beer, H., Walter, G., Macklin, R. L., and Patchett, P. J. 1984, Phys. Rev. C30, 464.

Blanco, V. M., McCarthy, M. F., and Blanco, B. M. 1980, Ap. J., 242, 938.

Boothroyd, A., and Sackmann, I.-J. 1988a, Ap. J., 328, 653.

Boothroyd, A., and Sackmann, I.-J. 1988b, Ap. J., 328, 671.

Brehm, K., Becker, H. W., Rolfs, C., Trautvetter, H. P., Käppeler, F., and Ratynski, W. 1988, Z. Physik A330, 167.

Burbidge, G. R., Burbidge, E. M., Fowler, W. A., and Hoyle, F. 1957, Rev. Mod. Phys., 29, 54.

Busso M., and Gallino, R. 1983, Ap. and Space Sci., 94, 273.

Busso M., and Gallino, R. 1985, Astron. Astrophys., 151, 205.

Busso, M., Picchio, G., Gallino, R., and Chieffi, A. 1988a, Ap. J., 326, 196.

Busso, M., Gallino, R., Käppeler, F., Picchio, G., and Raiteri, C. M., 1988b, Comparison of $s$-Processing in Low Mass AGB Stars with the Classical s Analysis, in preparation.

Cameron, A. G. W. 1955, Ap. J., 121, 1446.

Clayton, D. D. 1988, Nature, 332, 683.

Clayton, D. D., Fowler, W. A., Hull, T. E., and Zimmerman, B. A. 1961, Ann. Phys., 12, 331.

Clayton, D. D., and Rassbach, M. E. 1967, Ap. J., 148, 69.

Cohen, J. G., Frogel, J. A., Persson, S. A., and Elias, J. H. 1981, Ap. J., 249, 481.

Cosner, K., Iben, I., Jr., and Truran, J. W. 1980, Ap. J. Letters, 238, L91.

Couch, R. G., Schmiedekamp, A. R., and Arnett, W. D., 1974, Ap. J., 190, 95.

Cowley, C. R., and Downs, P. L. 1980, Ap. J., 236, 648.

Despain, K. H. 1980, Ap. J. Letters, 236 , L165.

Dominy, J. F., and Wallerstein, G. 1986, Ap. J., 310, 371.

Fowler, W. A., Caughlan, G. R., and Zimmerman, B. A. 1967, Ann. Rev. Astron. Astrophys., 5, 525.

Fuller, G. M., Fowler, W. A., and Newman, M. J. 1983, Ap. J. Supp., 329, 943.

Gallino, R., and Busso, M. 1985, in From Nuclei to Stars, ed. A. Molinari and R. A. Ricci (Amsterdam: North-Holland), p.309. 
Gallino, R., Busso, M., Picchio, G., Raiteri, C. M., and Renzini, A. 1988a, Ap. J. Lett., 334, L45.

Gallino, R., Busso, M., Picchio, G., Raiteri, C. M., 1988b, An Interpretation of $\mathrm{Ne}, \mathrm{Kr}$ and $\mathrm{Xe}$ Isotopic Anomalies in the Murchison and Murray Meteoritic Inclusions, 1988, in preparation.

Habing, H. J. 1987, in The Galaxy, ed. G. Gilmore and B. Carswell (Cambridge: Cambridge Univ. Press), p.173.

Hollowell D. E., and Iben, I., Jr. 1988a, in Atmospheric Diagnostics of Stellar Evolution, Proc. IAU Coll. no. 108 (Berlin: Springer-Verlag), in press.

Hollowell, D. E., and Iben, I., Jr. 1988b, Ap. J. Letters, 333, L25.

Hollowell, D. E., and Iben, I., Jr. 1988c, Neutron Production and Neutron-Capture Nucleosynthesis in a Low-Mass, Low-Metallicity AGB Star, preprint.

Howard, W. M., Mathews, G. J., Takahashi, K., and Ward, R. A. 1986, Ap. J., $309,633$.

Iben, I., Jr. 1975a, Ap. J., 196, 525.

Iben, I., Jr. 1975b, Ap. J., 196, 549.

Iben, I., Jr. 1976, Ap. J., 208, 165.

Iben, I., Jr. 1977, Ap. J., 217, 788.

Iben, I., Jr. 1982, Ap. J., 260, 821.

Iben, I., Jr. 1983, Ap. J. Lett., 275, L65.

Iben, I., Jr., and Renzini, A. 1982a, Ap. J. Lett., 259, L79.

Iben, I., Jr., and Renzini, A. 1982b, Ap. J. Lett., 263, L23.

Iben, I., Jr., and Renzini, A. 1983, Ann. Rev. Astr. Ap., $21,271$.

Jorgensen, U. G. 1988, Nature, 332, 702.

Jorissen, A., and Arnould, M. 1986, in Nucleosynthesis and its Implications on Nuclear and Particle Physics, ed. by J. Audouze and N. Mathieu (Dordrecht: Reidel), p. 303.

Käppeler, F. 1986, in Advances in Nuclear Astrophysics, ed. E. Vangioni-Flam, J. Audouze, M. Cassé, J. P. Chieze and J. Tran Thanh Van (Paris: Editions Frontières), p. 355.

Käppeler, F., Beer, H., Wisshak, K., 1988, private communication.

Käppeler, F., Beer, H., Wisshak, K., Clayton, D. D., Macklin, R. L., and Ward, R. A. $1982, A p . J ., 257,821$.

Klay, N., and Käppeler, F. 1988, Phys. Rev. C38, 295.

Krishnaswamy-Gilroy, K., Sneden, C., Pilachowski, C.A., and Cowan, J.J. 1988, Ap. J., 327, 298.

Lamb, S.A., Howard, W.M., Truran, J.W., and Iben, I., Jr. 1977, Ap. J., 217, 213.

Lambert, D. L. 1985, in Cool Stars with Excesses of Heavy Elements, eds. M. Jaschek and P. C. Keenan, (Dordrecht: Reidel), p.191.

Lattanzio, J. C. 1987, Ap. J. Lett., 313, L15.

Little, S. J., Little-Marenin, I. R., and Bauer, W. H. 1987, A. J., 93, 1539.

Luck, R. E., and Bond, H. E. 1982, Ap. J., 259, 792.

Malaney, R. A. 1986a, M.N.R.A.S., 223, 683.

Malaney, R. A. 1986b, M.N.R.A.S., 223, 709.

Malaney, R. A. 1986c, Advances in Nuclear Astrophysics, ed. E. Vangioni-Flam, 
J. Audouze, M. Cassé, J. P. Chieze and J. Tran Thanh Van (Paris: Editions Frontières), p. 407.

Malaney, R. A. 1987, Ap. J., 321, 832.

Malaney, R. A., and Lambert, D. L. 1988, M.N.R.A.S., in press.

Mathews, G. J., and Ward, R. A. 1985, Rep. Progr. Phys., 48, 1371.

Mould, J., and Reid, N. 1987, in Late Stages of Stellar Evolution, ed. S. Kwok and S.R. Pottasch, (Dordrecht: Reidel), p.209.

Ott, U., Begemann, F., Yang, J., and Epstein, S. 1988, Nature 332, 700.

Peery, B. F., and Beebe, R. F. 1970, Ap. J., 160, 619.

Peters, J. G. 1968, Ap. J., 154, 224.

Picchio, G., Busso, M., Gallino, R., and Raiteri, C. M. 1988a, in Mass Outflows from Stars and Galactic Nuclei, ed. L. Bianchi and R. Gilmozzi (Dordrecht: Kluwer Academic Publ.), p. 279.

Picchio, G., Busso, M., Gallino, R., and Raiteri, C. M. 1988b, The Neutron Source ${ }^{13} \mathrm{C}(\alpha, n){ }^{16} \mathrm{O}$ in Thermally Pulsing Stars and the s-Processing, preprint.

Prantzos, N., Arnould, M., and Arcoragi J.-P. 1987, Ap. J., 315, 209.

Raiteri, C. M., Busso, M., Gallino, R., Picchio, G., and Renzini, A. 1988, The Effect of Light $n$-Poisons on the $s$-Nucleosynthesis of AGB Low Mass Stars of Different Metallicity, in preparation.

Ratynski, W., and Käppeler, F. 1988, Phys. Rev. C37, 595.

Reeves, H. 1966, Ap. J., 146, 447.

Reffo, G. 1988, private communication.

Sackmann, I.-J., 1980, Ap. J. Lett., 241, L37.

Sanders, R. H. 1967, Ap. J., $150,971$.

Schwarzschild. M., and Härm, R. 1965, Ap. J., 142, 855.

Schwarzschild. M., and Härm, R. 1967, Ap. J., 150, 961.

Seeger, P. A., Fowler, W. A., and Clayton, D. D. 1965, Ap. J. Suppl., 11, 121.

Smith, V. V., and Lambert, D. L. 1985, Ap. J. , 294, 326.

Smith, V. V., and Lambert, D. L. 1986, Ap. J. , 311, 843.

Smith, V. V., and Lambert, D. L. 1988, s-Process Enriched Cool Stars with and without Technetium: Clues to AGB and Binary Star Evolution, Ap. J., in press.

Smith, V. V., Lambert, D. L., and McWilliam, A. 1987, Ap. J. , 320, 865.

Smith, V. V., and Wallerstein, G. 1983, Ap. J., 273, 742.

Spite, M., and Spite, F. 1978, Astr. Ap., 67, 23.

Sugimoto, D., and Nomoto, K. 1975, Publ. Astr. Soc. Japan, 27, 197.

Takahashi, K., and Yokoi, K. 1987, Atomic Data and Nucl. Data Tables, 36, 375.

Thielemann, F.-K., and Arnett, W. D. 1985, Ap. J., 295, 589.

Tomkin, J., and Lambert, D. L. 1986, Ap. J., 311, 819.

Truran, J. W., and Iben, I., Jr. 1977, Ap. J., 216, 797.

Ulrich, R. K. 1973, in Explosive Nucleosynthesis, ed. D. N. Schramm and W. D. Arnett (Univ. of Texas: Austin), p. 139.

Wallerstein, G., and Dominy, J. F. 1988, Ap. J., 330, 937.

Walter, G., Beer, H., Käppeler, F., Reffo, G., and Fabbri, F. 1986, Astron. Astrophys., 167, 186. 
Ward, R. A., and Newman, M. J. 1978, Ap. J., 219, 195.

Weigert, A. 1966, Z. Physik, 64, 395.

Winters, R. R., and Macklin, R. L. 1988, Ap. J., 329, 943.

Zook, A. C. 1978, Ap. J. Lett., 221, L113.

Zook, A. C. 1985, Ap. J., 289, 356. 